\title{
Germanica
}

\section{La mort en miroir. Religion et foi dans l'œuvre de Gerrit Achterberg}

De dood in spiegelbeeld. Godsdienst en geloof in het werk van Gerrit Achterberg

\section{Spiros Macris}

\section{Q OpenEdition}

\section{Journals}

Édition électronique

URL : http://journals.openedition.org/germanica/2050

DOI : 10.4000/germanica.2050

ISSN : 2107-0784

Éditeur

Université de Lille

\section{Édition imprimée}

Date de publication : 31 décembre 1996

Pagination : 83-116

ISSN : 0984-2632

\section{Référence électronique}

Spiros Macris, « La mort en miroir. Religion et foi dans l'œuvre de Gerrit Achterberg », Germanica [En ligne], 19 | 1996, mis en ligne le 04 juin 2013, consulté le 06 octobre 2020. URL : http://

journals.openedition.org/germanica/2050 ; DOI : https://doi.org/10.4000/germanica.2050

Ce document a été généré automatiquement le 6 octobre 2020.

(c) Tous droits réservés 


\title{
La mort en miroir. Religion et foi dans l'œuvre de Gerrit Achterberg
}

De dood in spiegelbeeld. Godsdienst en geloof in het werk van Gerrit Achterberg

\author{
Spiros Macris
}

\begin{abstract}
L'auteur se demande quelle sera la bonne méthode, l'ontologique ou la psychologique?

Flaubert, Bouvard et Pécuchet
\end{abstract}

1 Mettre en rapport le couple religion et foi avec la poésie n'est pas une démarche qui va de soi. Et cela à un double titre. Elle dépend, tout d'abord, de la perspective culturelle selon laquelle on l'aborde. Ainsi en France le critère ne paraît guère pertinent: un poète peut être croyant sans se dire "poète catholique " par exemple. Cela n'implique pas pour le chrétien le refus des valeurs - la muse saura garder son rang - mais plutôt la conscience qu'une pratique peut être libre tout en étant soumise. C'est aussi l'expression du fait que l'identité religieuse n'a pas besoin d'être définie, puisqu'elle n'est jamais confrontée qu'à elle-même. Ainsi posée, la question est fondamentalement de nature thématique.

2 Aux Pays-Bas la situation est presque l'inverse. La reconnaissance de l'identité et de la diversité religieuse, à l'origine de la nation même et de la culture hollandaise, conduit à la voir partout présente et partout acceptée sinon revendiquée. La culture commune s'en est imprégnée car sa constitution n'est pas issue d'une lutte contre le religieux mais de la recherche d'un équilibre, revendiqué par Guillaume le Taciturne dès l'origine, entre les identités : équilibre acceptable non parce que parfait ou juste, mais parce que toujours susceptible de changer. Le couple religion et foi est ainsi, même dans la société actuelle largement laïcisée, souvent un point de départ, voire un préalable, et cela du côté de l'auteur comme du lecteur. Ainsi «De conferentie» (1890), récit de l'écrivain limbourgeois $\mathrm{F}$. Erens, paraitra à un lecteur hollandais «quand même » très catholique, tandis qu'un Français trouvera l'œuvre typiquement impressionniste. Chacun décrit ainsi un rapport particulier aux formes et aux couleurs, à un mode d'existence plastique. Il ne s'agit ni de vocabulaire ni même de références 
culturelles autres, mais bien de façons différentes de s'inscrire dans un réel et, dans le même mouvement, de le constituer.

3 Mais la voie proposée comporte un autre trait particulier quant à son rapport au fait littéraire et, plus particulièrement, poétique. Poser le problème de la religion et de la foi, c'est poser le problème du rapport à l'autre, au groupe, à sa propre culture. Au rebours des acquis de la poétique moderne centrée sur le texte, l'extérieur fait ici irruption, le non-textuel s'insinue de toutes parts. L'objet scientifiquement constitué se défait et cède devant le mystère - quel qu'en soit l'origine.

Aborder de la sorte l'œuvre de Gerrit Achterberg conduit donc à tenir compte à la fois de la dimension thématique, qui s'offre à fleur de texte, mais également d'un principe de vérité, c'est-à-dire d'un monde, relatif dans son étendue et absolu dans son principe. $\mathrm{Au}$ passage d'un monde à un autre, les réalités que l'on pense identiques changent de signe et l'opposition fondamentale extérieur / intérieur, être "dedans " ou être "dehors ", se révèle être un axe fondamental. Il apparaît notamment dans la thématique, problématise la clôture du texte et impose un rapport au monde structurant l'ensemble. Toute étude littéraire, même si elle se concentre sur un seul auteur enraciné dans sa propre culture, devra toujours être, selon l'heureuse définition, générale et comparée.

5 L'importance du rapport au fait religieux est manifeste dans l'œuvre. Le poète entretient au début de sa vie des liens nombreux et étroits avec les milieux réformés et plus tard, tout en prenant ses distances, ne marquera jamais d'opposition au calvinisme. Cela prend d'autant plus d'importance que, surtout dans la deuxième moitié du siècle, mais déjà bien avant, la lutte contre l'emprise calviniste est au cœur d'œuvres nombreuses et importantes; la particularité de cette emprise étant de toucher au médium même de l'écrivain. Chacun connaît, aux Pays-Bas, l'expression "parler la langue de Canaan ». Cela signifie, par référence au livre d'Isaïe (19: 18), utiliser la langue spécifique des milieux réformés de stricte obédience, caractérisée par des tournures et un vocabulaire empruntés à la bible, selon la traduction dite des Etats (1637), et, plus généralement, par un esprit qui donne aux mots courants un sens spirituel particulier. Tout comme la rédaction de la Bible des États, éditée afin que l'on puisse entendre Dieu parler dans la langue néerlandaise ${ }^{1}$, a constitué une étape majeure dans la fixation du néerlandais, l'empreinte de cette langue sur le milieu par lequel le poète a été formé est très profonde. Il s'agit moins ici d'idéologie, bien que très présente, que d'une structuration fondamentale articulant langage et existence.

6 C'est à partir de cette donnée qu'il est possible d'envisager l'efficace de cette poésie. Cette efficace réside, sans doute, dans le texte; elle se déploie cependant dans un espace signifiant non-textuel et en tire sa puissance. La lettre reflue et se dissout dans un ensemble plus vaste. Le texte, comme objet, s'offre à l'analyse, et, dans le même mouvement, se dérobe comme poème. Cela vaut pour toute parole de poésie, mais ici l'horizon du poème est particulier dans le sens où la clôture du texte est mise en question par la définition de la parole. Pour rendre compte de l'attrait qu'exerce l'œuvre d'Achterberg, on pourrait, afin de rester au plus près de l'expérience de la lecture, comme d'ailleurs de la réaction d'une partie de la critique, retenir le terme de fascination. Présence élusive et obstinée, qui attire le regard et se dérobe tout à la fois.

7 La nature de l'œuvre de Gerrit Achterberg est donc telle qu'elle semble s'offrir de manière privilégiée à une approche par le fait religieux, et nombreux sont ceux qui ont emprunté cette voie. Tout les y invite. 
8 Le poète naquit en 1905 à Neerlangbroek ${ }^{2}$, petit village au sud-est d'Utrecht où il passa son enfance puis son adolescence jusqu'en 1924, date à laquelle il trouva un emploi d'instituteur dans une école réformée dans un bourg près du Rhin. La région connaissait un clivage social très marqué avec d'une part quelques grandes familles, propriétaires de domaines et de châteaux, et d'autre part une population composée de paysans, souvent métayers. Son père était le cocher du comte Van Lynden van Sandenburg, qui avait le pouvoir réel dans la commune, et la maison natale se trouvait dans le parc derrière l'orangerie. Les liens entre la famille Achterberg et celle du comte sont attestés dès le début du XIX ${ }^{\mathrm{e}}$ siècle. Vers la fin de sa vie, le poète se montrera curieux de ses origines et y trouvera une source d'inspiration.

9 La vie était dominée par la religion réformée, dont l'influence était considérable et qui réglait tous les aspects. L'accent était mis sur la communauté en tant que chacun, individuellement, devait ordonner sa vie, avec l'aide active du groupe si nécessaire, selon sa condition de pécheur. Le contrôle social va donc de pair avec le poids du péché originel porté par chacun, sans intermédiaire ecclésial - sola scriptura - mais également, selon les interprétations, sans certitude de salut. Les œuvres de l'homme ne comptent pour rien, il faut s'en remettre à la grâce divine. L'affirmation théologique de la toute puissance de Dieu reporte sur l'homme tout le poids de sa misérable condition. La tradition locale de la prédication était fort rigoureuse et même si, à l'époque où Achterberg fréquentait l'église, la dureté en était atténuée, les principes fondamentaux restaient inchangés. Que le jeune homme se soit bien intégré dans cette société pendant la première partie de sa vie est démontré par le fait qu'il était membre actif de Timotheus, une association réformée pour la jeunesse. Il le restera jusqu'à son départ $\mathrm{du}$ village natal et présentera, durant les réunions que les membres ouvraient et clôturaient par le chant de psaumes, des exposés consacrés essentiellement à des sujets religieux ou d'histoire nationale.

10 L'accent était mis sur la distance absolue existant entre Dieu, que l'on ne pouvait glorifier assez, et l'homme, le pécheur, qui devait être haï. Le livre de Job prenait une importance toute particulière car Job « était parfait et droit, craignant Elohim et se détournant du mal $»^{3}(1: 8)$ et s'il se repent et s'exècre, ce n'est pas à cause d'un péché précis (19:28), mais parce qu'il n'est qu'un homme devant son Dieu (42:6). L'homme est donc totalement impuissant et rien de ce qu'il pourra faire ne le rapprochera du salut : la prédestination est l'affirmation de cet abîme insondable.

11 Au fond de son désespoir, l'homme a pour seul appui et guide la Parole divine, sous la forme - pour la tradition qui nous occupe - définitive de la traduction réalisée sur l'ordre du synode de 1618 et 1619 , et avec l'orthographe ancienne pour les plus pieux. Le Verbe prend une place tout à fait particulière dans ce milieu. La réforme protestante a fait montre de défiance à rencontre de l'art en général et de la représentation en particulier. Le débat s'est ouvert bien avant le christianisme sans jamais s'apaiser tout à fait depuis. La position de Calvin sera bien plus radicale que celle de Luther et, sous la forme stricte adoptée par les réformés aux Pays-Bas, refusera tout ce qui ne vient pas de Dieu. Il n'y a qu'un seul texte et il doit être interprété littéralement. Tout le reste n'est que paille au vent. Le mystère de l'incarnation, de la Genèse à l'Apocalypse, tient tout entier dans le Livre, qui rend tous les autres superflues.

12 Le caractère abrupt de ces conceptions est toutefois adouci, au début de ce siècle, par l'invocation du Nouveau Testament : «Et il adviendra que quiconque invoquera le nom du Seigneur sera sauvé»(Ac. 2: 21). Ce salut prend notamment la forme d'une 
« régénération " (Jean $3: 7,1$ Pi. $1: 3$ ), pris au sens fort d'une «deuxième naissance ». Cette régénération n'est pas obligatoirement celle de l'âme après la mort, mais l'avènement de l'homme nouveau durant cette vie, sur cette terre. Le thème revient souvent dans l'œuvre et en constitue, sous la forme religieuse, une structure de base.

Pour aborder l'inspiration chrétienne du poète, le plus simple est de suivre ses propres indications et de se tourner vers le recueil En Jezus schreef in't zand [Et Jésus écrivit dans le sable], publié en $1947^{4}$. Le recueil est en fait une petite anthologie destinée aux lecteurs protestants et avait pour objet de toucher un publique plus large. Il n'est donc pas en tant que tel représentatif de l'œuvre. Il s'agissait d'obtenir une reconnaissance officielle, ce qui revêtait pour Achterberg une importance toute particulière sur laquelle nous reviendrons. Le poème éponyme tire son argument d'un passage de l'évangile de Jean sur l'épisode de la femme adultère (Jean $8: 111$ ) où il est dit par deux fois, sans autres détails, que Jésus « écrivait du doigt sur la terre ». Les deux premiers quatrains décrivent la scène puis :

Zondig niet meer, zei Hij, ik oordeel niet.

Ga heen en luister, luister naar het lied.

En Hij stond recht. De woorden lieten los

van hun figuur en brandden in een blos

waarmee zij heenging, als een kind zo licht.

Zo geestelijk schreef Jezus Zijn gedieht.

Ne péchez plus, dit-il, je ne juge pas.

Allez et écoutez, écoutez le chant.

Et Il se dressa. Les mots se détachèrent

de leur forme et brûlèrent en une rougeur

avec laquelle elle partit, légère comme une enfant.

Si inspiré était Jésus lorsqu'il écrivit son poème. ${ }^{5}$

Nous apprenons ainsi que c'est un poème que Jésus écrivit du doigt dans le sable. Ces trois strophes illustrent combien les principes poétiques s'expriment aisément en termes bibliques et paraissent même s'y fondre tout à fait. Les deux premiers vers de la citation reprennent presque littéralement le verset $8: 11$, complétant le discours du Christ par cette recommandation : « écoutez le chant. » C'est ensuite, en employant des termes concrets pour dire des choses abstraites, procédé familier au lecteur de la Bible surtout dans les traductions protestantes et caractéristique de l'œuvre d'Achterberg, l'opposition entre la lettre et l'esprit. Cette opposition entre la lettre qui tue et l'esprit qui est vie, selon la formule paulinienne ( 2 Cor. $3: 6$ ), est reprise dans le deuxième vers de cette strophe de manière tout aussi concrète : «brandden in een bios» rappelle en premier lieu le buisson ardent («het braambosch brandde in een vuur» Exo. $3: 2$ ) puis, avec l'enjambement, évoque les joues empourprées de la femme adultère qui retrouve son innocence d'enfant et enfin, par la rencontre entre le feu spirituel, un individu et le contexte de la Loi Nouvelle, l'effusion de l'esprit lors de la Pentecôte. Il a d'ailleurs consacré un poème («Pinksteren», 1940) à cet événement en suivant, comme ici, tout d'abord de près le texte des Actes. Dès le premier tercet cependant, on lit : «Onder het samenkomen / van klank en wezen moeten / zin en begrip verdwijnen» [Dans la rencontre / entre son et être / phrase et notion doivent disparaître]. On voit que poétique et religion cohabitent et que la première s'accommode fort bien des dehors de la seconde.

Ces rapports apparaissent fréquemment tout au long de l'œuvre. Voici, tiré du même recueil, un autre poème où ces liens se nouent de manière beaucoup plus complexe : 


\section{Triniteit}

God scherpt Zijn wet op deze steen, die mijn bestaan geworden is.

Maar Jesus geeft ons vis

en wijn tot Zijn gedachtenis.

Heeft Een van Beiden Zieh vergist?

Wij zijn een duister fenomeen,

zolang niet in ons leven rijst

het licht van den Heilige Geest.

Heilige Geest, kom in het vers,

waarin Gij Drieën, Een voor Een,

hetzelfde zijt en ik alleen

zingend van $\mathrm{U}$ de woorden ben.

Heilige Geest, vervul de vers

zo gans, dat er geen vezel is,

die niet van Uw belevenis

vibreert, als van de liefde vlees.

Moeder van Jesus is het vlees.

Zuster van Christus is het vers.

Vader, die in de Hemelen zijt.

kome Uw koninkrijk.

Trinité

Dieu aiguise Sa loi sur cette pierre,

qu'est devenue mon existence.

Mais Jésus Christ nous donne du poisson

et du vin en Sa mémoire.

L'Un des Deux S'est-Il trompé ?

Nous sommes un phénomène obscur,

tant que dans notre vie ne s'élève pas

la lumière du Saint Esprit.

Saint Esprit, entrez dans le poème,

dans lequel Vous Trois, Un par Un,

êtes le même et moi seul

chantant suis de Vous les paroles.

Saint Esprit, comblez le poème,

à tel point, qu'il n'y ait pas une fibre

qui de Votre expérience

ne vibre, comme de l'amour la chair.

Mère de Jésus est la chair.

Sœur du Christ est le poème.

Père qui êtes au cieux,

Que votre règne arrive.

Le mystère de la Trinité symbolise à lui seul l'essence du christianisme. Incarnation et rédemption marquent le passage de l'Ancienne Loi à la Loi Nouvelle et inaugurent pour tout chrétien l'espoir d'une renaissance. C'est là le point de départ de ce poème. Au dieu inflexible de l'Ancien Testament (v. 1, 2) est opposé le Christ. On s'attend naturellement à une évocation de la Cène et de l'instauration de l'eucharistie ; or, et on se trouve bien là en terre protestante, il n'y a pas de transsubstantiation mais une simple commémoration de l'événement. En outre, la présence du poisson, outre sa valeur symbolique, rappelle plutôt le déjeuner pris après la résurrection sur les bords de la mer de Tibériade (Jean $21: 13$ ).

La deuxième strophe constitue un tournant et la question du vers 5 interrompt toute velléité de poursuivre l'interprétation dans un sens théologique traditionnel. Cette 
coupure s'explique moins par le fait d'isoler les Deux premiers qui n'ont pas l'air de S'en sortir, que par le déplacement de la réalité du Saint Esprit dans le cadre de l'être humain. La visée n'est pas téléologique. Le glissement qui s'opère ici commande les rapports entre poésie et religion. Comme dans un corps pétrifié dont la substance organique est remplacée par la silice mais dont la forme reste parfaite, le principe poétique s'infiltre dans l'armature chrétienne et s'en rend maître. L'horizon est l'individu et le lieu de l'avènement de l'esprit est le poème (str. 3 et 4).

Ces deux strophes décrivent, en termes religieux, la structure fondamentale de la poésie d'Achterberg. Celle-ci emprunte ailleurs les formes les plus variées, ce qui conduit à distinguer l'élément permanent - la poésie - de ses figures successives, dont les attributs chrétiens font partie. Le poème est constitué par un matériau linguistique qui, pris dans une forme déterminée, convoque une présence dont l'identité est variable. Dans ce processus le poète occupe une position périphérique qui peut même être vécue comme passive. Cette position excentrée s'exprime ici par la réduction du Je à n'être ici que les mots de l'Esprit $(v .11,12)$ alors que la présence trinitaire est la forme parfaite d'un destinateur / destinataire omniprésent tout au long de l'œuvre.

Le Je n'existe - n'accède à la conscience - qu'au moment où le poème se fait et ne se définit que comme incarnation, c'est-à-dire une réalisation textuelle concrète, de la Parole divine. On retrouve ici la réalité même de la Bible : le Dieu infini doit - pour se faire connaître de l'homme - toujours et nécessairement s'exprimer de manière particulière, circonscrite. Texte absolu et circonstanciel, la Bible est un archétype du poème. Il est possible que la problématique si spécifique de la foi chrétienne soit liée à l'évolution du statut de l'écrit. Dans le passage où il évoque l'opposition entre la lettre et l'esprit, Paul mentionne d'ailleurs explicitement des techniques d'écriture : encre et gravure dans la pierre (2 Cor. $3: 3)$. Cela expliquerait : l'intégration du lien personnel avec Dieu, similaire à celui que suppose le rapport au texte littéraire; les rapports souvent homothétiques que l'on constate entre la littérature et des éléments clés du christianisme ; et, peut-être, les liens entre le protestantisme et la modernité depuis la Renaissance.

Dans «Triniteit», il s'agit bien pour le poète d'être ces mots et non d'en être le truchement. L'enjeu est ici l'identité, le mode d'existence, de la figure du poète et la force vive est du côté de la poésie, même dans un poème apparemment aussi " orthodoxe ». L'étude des variantes ${ }^{6}$ montre d'ailleurs que dans la première édition du poème, sous le titre «Bekering II» [Conversion II] dans le recueil Sintels [Scories] de 1944, le vers 12 était «nog zingende van U verschil» [chantant encore de Vous diffère]. Il s'agit donc bien d'emprunter des éléments à la religion en les détournant au profit de la poésie.

21 Dans le même recueil, le poème "Code " précise que l'énergie vitale, désormais dispersée par la mort, réside dans l'alphabet et peut être captée par le mot «Dieu ", composé des lettres $\mathrm{d}, \mathrm{i}, \mathrm{e}, \mathrm{u}$, «in deze volgorde, maar niet per se» [dans cet ordre, mais pas nécessairement] et que

Iedere serie, elke schakeling, uit welke taal genomen, is geschikt, zolang ze in de juiste spanning Staat. Toute série, tout enchaînement, pris dans n'importe quelle langue, convient, pourvu qu'il ait la tension exacte. 

entre les termes initiaux et décrit une Trinité nouvelle. Que la mère de Jésus soit la chair reflète sans doute la position des protestants vis à vis de l'Immaculée Conception et Achterberg sait être concret comme lorsqu'il parle de «de donkere eierstok / van ongeboren poëzie.» [l'obscur ovaire / de la poésie qui n'est pas encore née] ( Radium »), mais, plus profondément, pose le monde dans lequel nous vivons comme un des deux pôles créateurs, l'autre étant, ici, le Saint Esprit. A l'image de la première strophe, la dernière est également composée de deux parties. Mais les deux derniers vers, de nature traditionnelle, changent à présent de sens : le royaume des cieux, que le poète appelle de ses vœux, est celui de l'accomplissement de la poésie, selon la nouvelle Trinité. Le poème - c'est-à-dire la poésie réalisée - occupe donc une position identique à celle du Christ. Tous deux sont une parole en acte, efficace ici et maintenant, ayant partie liée avec la présence. Cette réalisation in concreto du poème, comme nous le verrons, n'est ni un retrait du réel dans quelque monde imaginaire ni l'expression d'une foi naïve dans la magie des mots en eux-même, mais la transcription par les moyens disponibles (détournement d'images, recomposition de symboles) de l'expérience poétique qui est celle de l'auteur au moment où le poème se constitue.

Publié dans Sneeuwwitje [Blanche Neige] en 1949, «Verzoendag» [Le jour de réconciliation ou de pardon] est un sonnet quasi régulier, les deux tercets se terminent sur des rimes suivies, basé sur un pentamètre iambique. Il témoigne ainsi de l'un des aspects marquants de l'évolution de l'œuvre dans le sens d'une maîtrise de plus en plus grande du rythme et de la forme, alors que la thématique reste par ailleurs remarquablement constante. Le sonnet notamment, avec ses diverses variantes, tend à dominer et Achterberg concevra durant les années cinquante plusieurs cycles.

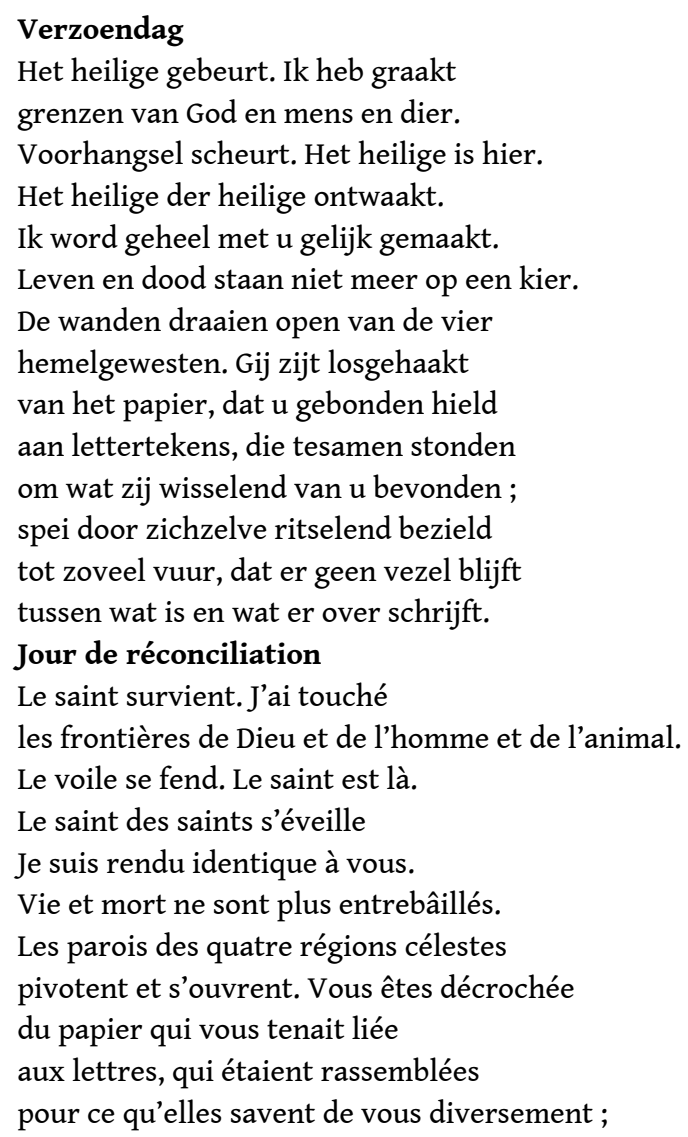


jeu par soi-même animé en bruissant

jusqu'à tant de feu, que nulle fibre ne reste

entre ce qui est et ce qui écrit là-dessus.

maintenant. Elle est par essence totale. La mention des «frontières de Dieu et de l'homme et de l'animal » ouvre l'espace entier en confondant créateur et créatures et arrête le temps. devient égal mais surtout identique, et dans ce moment anhistorique causes et effets se mêlent. Le poème se situe au partage des eaux entre la vie et la mort. Si elles ne sont plus séparées (v. 6), c'est parce que le poème participe des deux réalités à la fois au moment où l'esprit, la présence, naît de la tension particulière unissant les éléments constituants ayant partie liée avec la mort, l'inorganique. On reconnaît là un avatar du mystère de l'incarnation, mais on sait qu'entre l'argument et ce qui le porte, les liens ne sont pas toujours univoques.

La fin du huitième vers occupe une position particulière dans la progression du poème. Position soulignée par une irrégularité métrique, trois accents forts se suivent, et par l'enjambement entre quatrain et tercet. Cet enjambement relie deux domaines différents dans la poétique d'Achterberg. Jusqu'à la fin du quatrain on peut encore croire la place centrale occupée par le Christ: il s'agirait d'une simultanéité entre la mort sur la croix et la résurrection, manifestation de cette verticalité souvent évoquée en la matière. Mais dès le vers 9 , en l'espace d'un enjambement, on entre au cœur de ce qu'il est convenu d'appeler le « thème central » du poète. 

l'auteur et lui-même a légitimé cette approche. Ce thème se résume ainsi : la femme aimée n'est plus et le poète cherche à la faire revenir de cet ailleurs qu'est la mort. On pense naturellement à Orphée sauvant Eurydice des Enfers. Ici les Enfers sont la mort, ni lieu mythique, ni néant, mais: dispersion, perte de la forme, transformation moléculaire, mutation d'énergie, présence immanente : «Gij zijt geheel uiteen gegaan en liet / geen punt centraal [...]» [Vous êtes totalement éparpillée et n'avez laissé / nul point au centre] «Doodbeeld» [Image de mort (néo.)], ou encore :

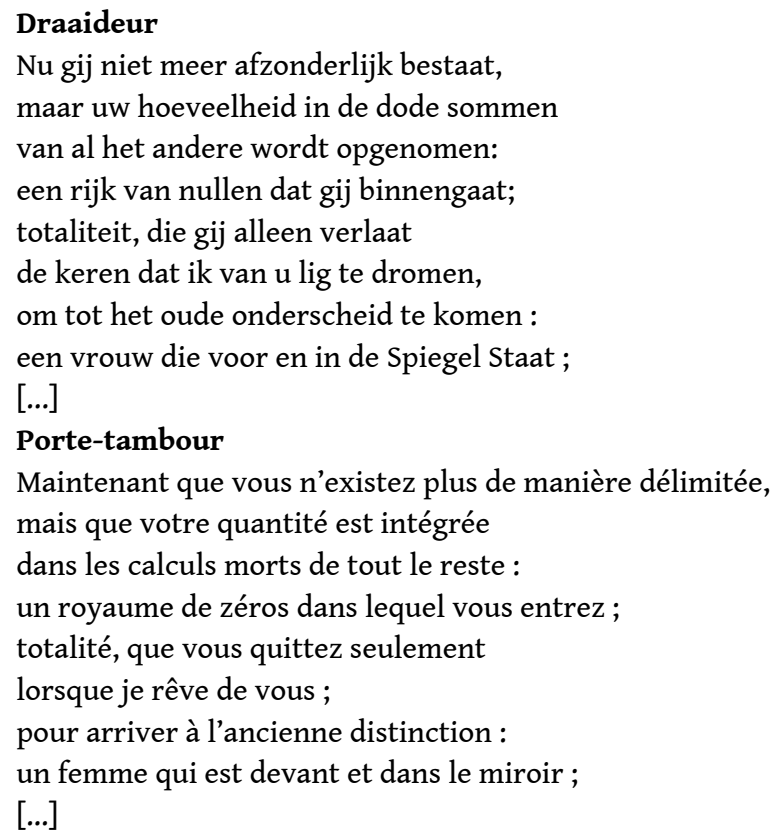

Contre cette dispersion, ce mode d'être inorganique, le poète dispose de la force structurante, organisatrice du langage. "De levenskracht die gij eenmaal bezat / verdeelt zieh nu over het abc» [L'énergie vitale que vous possédiez jadis / est répartie à présent sur l'abc] («Code», v. 1,2) : le poète peut donc rassembler le disséminement par les moyens de la poésie dont les limites sont celles de leur capacité structurante. La Bible offre en la matière des possibilités considérables, puisqu'elle va du chaos à la Parole, mais elle est loin d'être la seule voie. Tout ce qui lie l'épars offre une voie au poème.

Le thème central constitue l'argument d'une part importante de l'œuvre. Il est le point commun d'inspirations diverses. Etant protéiforme et réductible à un schéma quasi abstrait, le passage de l'inorganique à l'organique innerve toute l'œuvre. Cette double caractéristique prime les apparences successives quant au principe de cette poésie et s'efface dans chaque réalisation particulière. Que l'on puisse la plupart du temps lire dans ce thème des variantes de l'histoire d'Eurydice tient d'une part à un arrière-plan biographique et d'autre part au fait que le destinataire se manifeste le plus souvent sous la forme du pronom vous sans aucun réfèrent. Le traducteur français se trouvera d'ailleurs confronté au problème d'avoir à choisir un genre et n'aura parfois pas d'autre solution que d'invoquer l'hypothétique Eurydice.

Le thème apparaît dès les premiers poèmes dans toute sa netteté. Cette première occurrence, le poème a été publié en 1928, montre bien, sous la forme simple d'une triple union désignant traditionnellement le mariage, la tournure inattendue que peut 
prendre le thème. L'amour préservé, ce bonheur enfin réalisé, est obtenu grâce à la mort qui garantit l'apaisement des souffrances.

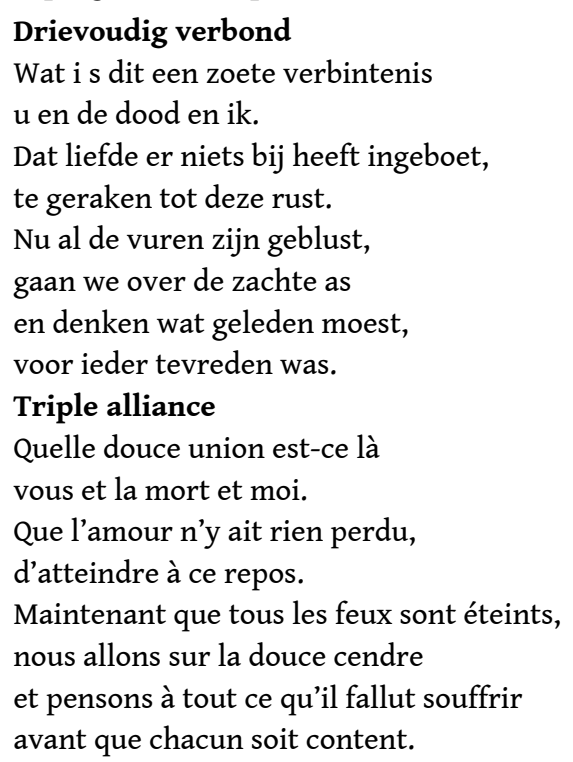

Il est encore présent dans « Anti-materie » [Antimatière], le dernier poème, achevé peu avant la mort du poète en 1962. Dans ce long poème la figure du Je, présence fantomatique, constate les traces disparates de sa vie en suivant les marques d'usure et les empreintes dans la pièce où il a vécu. Parmi les objets qui sont autant de témoins : «Een blad zit omgevouwen waar / je bent in het gesloten boek» [Une page est cornée là / où tu es dans le livre fermé]. Le passage, entre ces deux citations, d'une femme à un livre est significatif.

Cette référence au livre, à l'écrit, au poème occupe une place centrale car c'est là le point de rencontre entre la vie et la mort, le principe commun entre l'humain et le divin. Il y a une prééminence de la parole que le contexte culturel explique mais dont l'importance dépasse largement ce cadre. Si la poésie peut focaliser cette instance immanente, c'est qu'il y a un principe commun dont le Christ, Parole incarnée, n'est qu'une figure.

C'est bien ce dont il s'agit dans les tercets de «Verzoendag» : l'irruption du divin dans la poésie, superposé à l'image de la résurrection. On retrouve l'Esprit se dégageant de la lettre. Mais dans le passage du vers 8 au vers 9, bien que les forces en jeu restent les mêmes, le cadre de référence change. Les signes inconstants et fractionnés, petite monnaie des échanges quotidiens, ont trouvé dans le poème "la tension exacte » et c'est le destinataire, le Vous, qui se détache des mots. Dans le mouvement même de l'irruption, le poème se constitue en tant qu'objet réel. Ainsi le mythe chrétien prend l'allure d'un principe poétique et l'objet que le Vous représente s'élargit sans fin. Son indétermination grammaticale lui confère l'allure d'un support opalescent où l'on reconnaît sans cesse, et toujours avec raison, des femmes attestées par la biographie, des figures mythologiques, le Christ, le poème et enfin, par une réversibilité propre à ce rapport obstinément binaire, la figure du poète lui-même, comme le montre «Triniteit». Il ne s'agit pas là de la projection d'une identité nouvelle, mais d'un rapport où les deux pôles de la force créatrice garantissent mutuellement leur existence, leur réalité. Tel est le sens du dernier vers : au moment où le poème advient en tant que poème, où l'écheveau infini des sens dispersés prend consistance individuelle, le poète rejoint son objet: s'instaurent deux réalités coextensives dont la portée n'est pas 
circonscrite au support à l'origine de la concrétisation. Dans ce vers, le Je est aboli pour laisser place à « ce qui est » aucunement distinct de « ce qui écrit là-dessus ».

«Verzoendag» montre l'imbrication des éléments religieux et poétiques et le passage sans solution de continuité de l'un à l'autre. On pourrait multiplier les exemples : dans "Mania religiosa ", poème consacré à un fait divers, on lit : «En bijbelteksten lagen op de loer» [Et les textes bibliques étaient à l'affût]. Épisodes, lieux et personnages, citations : les Ecritures sont abondamment sollicitées. Mais l'accent est mis sur le mot, le langage, les symboles qui sont des instruments destinés à contraindre la lettre inessentielle à la présence.

La vertu du mot, porteur de sens, est de créer de l'ordre, de rassembler ce qui est dispersé. Il n'y a pas de domaine privilégié : le champ poétique couvre la totalité que l'homme porte en lui et dont il tire sa réalité. Cette extension englobe tout ce qu'une formulation retient dans ses rets. Si « la poésie a le secret du mal dont souffre l'être parlant » - la formule est d'Yves Bonnefoy - l'accent est ici placé sur " l'être parlant » et le poème peut survenir partout où il $\mathrm{y}$ a parole. Ce qui s'exprime chez Achterberg par une capacité permanente d'association. Tout peut être matière à poème car tout renvoie à la même réalité poétique centrale.

Le constat «Een goddelijk bevel / is overal» (v. 15-16) [Un ordre divin / est partout] pourra donc trouver sa place dans un poème, «Oppervlaktespanning» [Tension superficielle], dont le schème organisateur est d'ordre scientifique. La tension superficielle est une «force, due aux interactions moléculaires, qui s'exerce à la surface d'un liquide au contact d'un autre fluide (exprimée en dynes, agissant sur un élément linéaire de $1 \mathrm{~cm}$ de longueur) " (Le Robert). Elle détermine donc un mode d'être involontaire mais omniprésent qui, à ce titre, régit un mode de création, et ce doublement: comme mode de production inconscient de l'autre et comme processus poétique. La fondamentale du poème est le thème central : «Étendu, je pense à vous dans la mort» (v. 1). La loi physique induit alors deux mouvements. Après que la "tension superficielle de ma volonté » (v. 2) a immobilisé l'univers et ses forces, l'inconscient du Je s'extravase vers le Vous (v. 10-14):

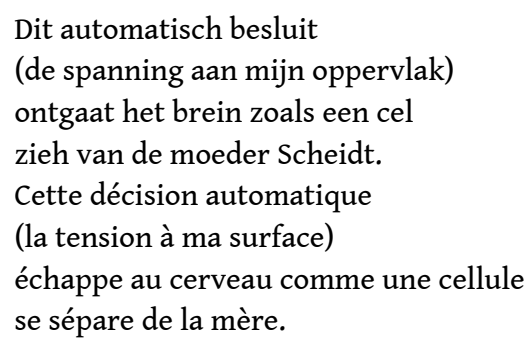

Cet insu ouvre un espace échappant à la conscience dans lequel naît et croît un être nouveau, commençant en "grappes de principe de vie» (v. 19), cherchant «centre et sens » (mais «zin» outre « sens » signifie également « envie » et surtout « phrase »)

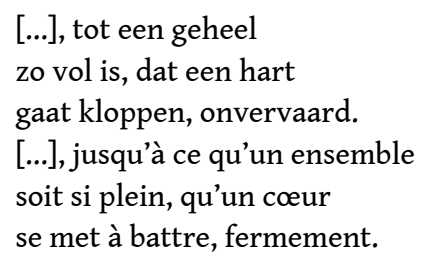

Cette force définie par la science devient un auxiliaire pour le poète. C'est une occasion de plus pour rendre l'aimée présente sous une forme thématisée et, du point de vue de la poétique, un instrument supplémentaire pour secourir l'être parlant, lequel n'est pas 
absolument dénué de connaissances. Cette double fonction explique à la fois la prégnance du thème central et la difficulté à le définir. En dernière instance il se confond totalement avec le projet poétique.

L'encyclopédie est naturellement une source d'inspiration privilégiée. De ce point de vue elle ressemble à la Bible. Il ne s'agit pas seulement d'un réservoir d'images et de thèmes - de ce point de vue les deux textes sont inépuisables - mais aussi de voies d'accès structurées et structurantes au réel, au grand inconnu dans lequel baigne l'être parlant. Il existe naturellement d'autres guides : arithmétique, religions anciennes, contes traditionnels, folklore, médecine, événements du quotidien, instruments divers etc.

Le poème " Proteine ", publié en revue en 1947 et repris la même année dans le recueil Doornroosje [La belle au bois dormant], est un exemple typique de cette progression partant d'une donnée scientifique. Le poème commence ainsi : «Achtien aminozuren waren / het eiwit, waarmee gij begon, / lees ik in deze lexicon.» [Dix-huit acides aminés constituaient / la protéine, par laquelle vous avez commencé, / c'est ce que je lis dans cette encyclopédie.] L'approche scientifique, les décomptes et les statistiques offrent un moyen de pénétrer plus avant dans l'intimité de la vie et sont donc autant d'images du travail du poète.

L'espace euclidien, la pression osmotique, la dévaluation sont des principes et des forces qui établissent un lien entre les mots et le réel. Ce sont des métaphores de la création poétique et Achterberg les utilise en tant que tels. Ce sont également des éléments objectifs, attestés par leur utilité, et arbitraires qui sont à même de déterminer la formation d'un poème faisant fond sur une réalité extérieure et induisant un sens nouveau. Le poète y trouve un point d'appui pour reconstruire un Je toujours menacé par l'abandon total qu'exige le poème. L'individu est mis en suspens pour n'être plus qu'une pure attente, une disponibilité totale.

Cet abandon semblerait en premier lieu le signe de la totale confiance dans la parole en tant qu'existant absolument. Ce serait cependant une erreur que d'y voir l'expression d'un panlogisme. «Tekort» [Carence], publié dans le recueil Dead End (1940), déplace ainsi vers la substance du poème le centre de gravité du poète qu'il définit ainsi dans la première strophe :

Ik ben het bitter overschot, aan deze zijde Gods, van ons in u voltrokken lot. Je suis l'amère dépouille, en deçà de Dieu, de notre destin en vous accompli.

La dernière strophe, composé d'un seul vers, est la reprise presque littérale du message johannique: "Het woord was God in den beginne.»[La Parole était Dieu au commencement]. Cette affirmation n'est pas théologique mais pose un horizon poétique à la dimension de l'être parlant et qui délimite le champ clos dans lequel se déroule le "combat à la mort et à la vie» ("Code», v. 13). Dégagée du contexte religieux, elle trouve un équivalent exact dans Benveniste: "Le langage est dans la nature de l'homme, qui ne l'a pas fabriqué. [...] le langage enseigne la définition même de l'homme. $»^{8}$ En changeant de contexte la proposition garde la même polarité : l'homme ne «fait » toujours pas le langage, mais Dieu se confond avec l'aimée, avec ce Vous s'élargissant à l'infini, et sa puissance est répartie entre les lettres de l'alphabet. 
Cette virtualité n'exclut en rien les risques du processus poétique. Le mot n'a pas de vertu intrinsèque :

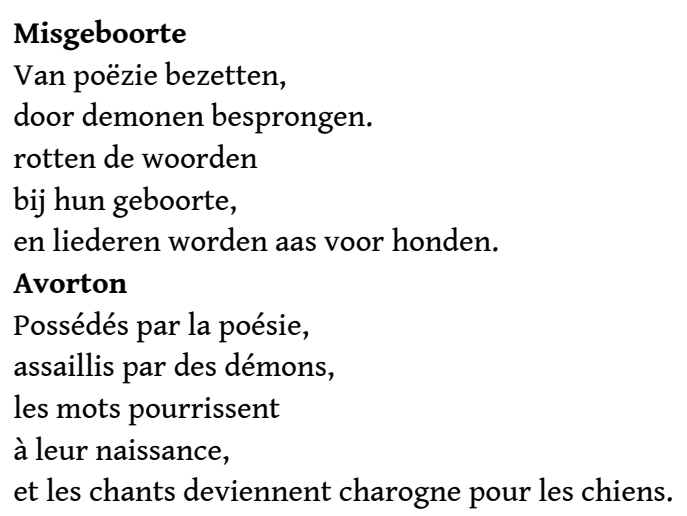

L'avènement du destinataire, le Vous omniprésent, et donc du poème, n'est garanti que par l'énergie resserrée dans la forme du poème lui-même, rimes et sonorités, mètre liant les images, constitution d'une nécessité illuminant le réel. Auxiliaires précieux, médiateurs puissants. Les constats sur lesquels prend appui le poème, se nouent hors de lui. D'où l'importance de la Bible ou de l'encyclopédie et de la place que prennent chez Achterberg l'association d'éléments. Ce sont des points d'ancrage pour ces «wetten [...]/ der waarheid, die gij zijt / binnen het alfabet. » [lois [...]/ de la vérité que vous êtes / à l'intérieur de l'alphabet] («Verzet» [Résistance]).

La mort, thème si fréquent dans ces poèmes, n'est pas le terme d'un processus, mais une présence constante. Une sorte de monde parallèle d'où proviennent des appels et qui devient réversible si le temps linéaire est suspendu, par la poésie par exemple.

\section{Ik wist niet...}

Ik wist niet dat hij al sprak in de taal van de dood.

Hij zei: vannacht ben ik weer geweest,

waar je je naam in Spiegelschrift leest,

een armlengte boven je hoofd.

Je ne savais pas...

Je ne savais pas qu'il parlait déjà la langue de la mort.

Il dit : cette nuit je suis venu de nouveau,

là où tu lis ton nom dans une écriture en miroir,

à un bras au-dessus de ta tête.

Dans ces quatre vers sont réunis un Je spectateur, la mort comme source de parole, une langue mystérieuse et pourtant compréhensible, succession d'observations incontestables et logiques dont la somme reste incomplète. Chaque élément est concret mais, tout en s'imposant, pointe vers ce qui est tu, comme dans un rêve.

Le sens circule ainsi de constat en constat se heurtant à des nœuds de significations parfois opaques dont les référents sont soit déplacés soit absents. Le poème est un pont dont l'un des jambages reste en suspens. Ce suspens - c'est, de nouveau, ce qui se dérobe dans le langage - peut, en première instance, s'analyser comme un rapport à la création poétique dominée par un état réceptif total qui place le poète en position seconde et projette le poème dans l'avenir. Le poème qui suit, publié en 1936 ou 1937, regroupe quelquesuns des thèmes majeurs. Il montre à quel point l'élément chrétien fait partie d'une circulation du sens s'établissant autour l'avènement, contre la mort, du poème. On retrouve les éléments constituant «Triniteit» et «Verzoendag», mais comme soumis à une permutation. Le rapport au divin devient un débat intérieur; le Christ et la Trinité, Vous; le règne du Père, le poème. 
Met dit gedieht...

Met dit gedieht vervalt het vorige.

Ik blijf mijn eigen onderhorige.

Totdat in 't einde blijken zal,

wie meester is, en wie vazal.

Tussen mijn leven en mijzelve

is enkel nog een graf te delven.

Maar buiten deze laatste dingen

is enkel nog het lied te zingen,

- is enkel nog den dood t'ontwringen

het lied dat van haar lichaam is,

het lied waarvan haar lichaam is

de onbevlekte ontvangenis

en dat den dood niet toebehoort

binnen dit woord.

Par ce poème...

Par ce poème le précédant est périmé.

Je reste mon propre subordonné.

Jusqu'à ce qu'à la fin se révélera,

qui est le maître et qui le vassal.

Entre ma vie et moi-même

il n'y a plus qu'une tombe à creuser.

Mais hormis ces dernières choses

il n'y a plus que le chant à chanter,

- il n'y a plus qu'à arracher à la mort

le chant qui est de son corps

le chant dont son corps est fait

l'immaculée conception

et qui n'appartient pas à la mort

à l'intérieur de cette parole.

On reconnaît le thème structurant du rapport Je - Vous; la position centrale de la mort; le poème, comme corps structuré, lieu de la sauveté, manifestant la capacité préservatrice de la parole.

Le premier vers résume ce qui est peut-être la grande vérité d'Achterberg et qui définit sa poétique comme sa vie au quotidien : rien n'existe si ce n'est le poème en cours. Tous les précédents sont devenus des objets sociaux et utilisés dans ce cadre; tous ceux qui sont à venir sont l'espoir qui maintient le poète en vie et il ne faut pas voir dans cette formule une simple image. Cette vérité régit sa vie au point qu'elle devient un leitmotiv dans sa correspondance. La phrase : «Wat niet goed is, is niet geschreven. Ik hoop te mögen hopen op het vers» [Ce qui n'est pas bon, n'est pas écrit. J'espère pouvoir espérer le poème] est devenue le véritable symbole de sa manière d'être. Suit immédiatement (v. 2) ce qu'il faut bien considérer comme un corollaire : le clivage du Je. Le poète se définit comme une pure attente du poème à venir, venue sur laquelle il n'a d'autre prise que d'en augmenter ses chances grâce aux outils que sont les traités et autres dictionnaires et en se délestant de tout autre préoccupation, y compris même, dans une certaine mesure, l'intendance de l'œuvre : sélection de poèmes et constitution des recueils, recherche de titres etc. Se dire à la fois dominé et dominant, c'est définir cette attente comme un lieu de passage, ou plus exactement de transmission entre ce qui se manifeste dans la mort, ce monde proche et étranger, et ce qui est préservé dans le poème par la lutte du poète. existence biographique. Ce qui fait l'existence du poète, n'est réalisé que par les mots 
qui, tirés de cette mort destructurante, accèdent à une réalité partageable grâce à une forme porteuse de tension, et donc à même de trouver la reconnaissance sociale.

Tel est l'unique sujet d'Achterberg: le passage de la mort au poème ; c'est-à-dire de la dissolution contre laquelle tente de s'établir la force qui lie les éléments physiques et psychiques qui nous composent, à la structure individuée et permanente d'éléments linguistiques et psychiques qui composent le poème. Dans une lettre du 4 janvier 1948, il se défend contre un critique paraissant prendre au pied de la lettre l'idée que le poète tente d'insuffler la vie à la matière inerte. A cette " plaisanterie » il répond : «Ik bedoel zooiets als: In de levende schoonheid van het vers is de dood als zodanig opgeheven» ${ }^{9}$ [Je veux dire quelque chose comme : Dans la beauté vivante du poème, la mort en tant que telle est suspendue]. Il faut donc que le poète ait partie liée avec la mort puisqu'il participe de la dispersion des forces tout en focalisant celles-ci. L'importance des métaphores techniques (radiotélescope, soudure autogène, radiographie etc.) témoignent de cette instrumentalisation du poète.

Le vers 7, opérant la transition, montre bien que dès le poème formé, ou en formation, la réalité est régénérée et acquiert profondeur. Le poème devient sujet et objet à la fois. L'immaculée conception - si peu protestante - ne renvoie pas à la création du poème mais au fait que la mort en est réellement absente. Le scheine religieux est ici mis au service de la poésie. Il rétablit l'identité entre le poème, Vous (destinataire implicite) et le Christ. Ce dernier, présent par la référence à sa naissance comme par le rappel que la parole échappe et s'oppose à la mort (Jean $5: 24$ ), est comme le témoignage redoublé de la permanence du poème.

55 Le Je est une pure instance de poésie, car dans le processus de création et dans sa préparation, le substrat biographique, se réduit à n'être plus qu'une ombre à toutes superposable. Reste à concentrer dans le mouvement infini du sens fractionné, les éléments structurants: «Woorden, ontwaak, ik ben uw naam, / ik ben uw enige bestaan. / Ik zie mij ongeboren aan.» [Mots, éveillez-vous, je suis votre nom, / je suis votre unique existence. / Je m'observe pas encore né.] Presque chaque poème aborde l'objet central de cette quête, en y intégrant l'intensité de ses rapports amoureux, en empruntant aux nœuds de signification du réel, quel qu'en soit le sujet, ou bien encore en mettant en scène cette quête dans des décors différents. Un exemple célèbre de ce dernier mode est «Farao» (1939) où le Je - pharaon énumère ce qui doit être placé dans sa tombe et termine par ces mots : «maar leg als laatste wat gij doet / al mijn gedichten aan mijn voet; / krachten, waarmee ik opstaan moet.» [mais que la dernière chose que vous déposiez / soit tous mes poèmes à mes pieds; / forces, avec lesquelles je me lèverai.]

Dès 1939, dans «Grafschrift» [Epitaphe], le quatrain sera effectivement placé sur sa tombe, le poète avait résumé ainsi son destin :

Van dood in dood gegaan, totdat hij stierf.

De namen afgelegd, die hij verwierf.

Behoudens deze steen, waarop geschreven:

de dichter van het vers, dat niet bedierf.

Passé de mort en mort, jusqu'à ce qu'il meurt.

Déposé les noms qu'il avait acquis.

A l'exception de cette pierre, où est écrit :

l'auteur du poème qui jamais ne se corrompit.

57 La nécessité de lier la thématique littéraire à un ensemble plus large nous était suggérée par le choix d'aborder l'œuvre par le biais de la religion. Certes, aucune œuvre 
n'est refermée sur elle-même : le monde est toujours convoqué et l'œuvre littéraire, quoique qu'ait pu en dire la critique, s'y enracine profondément. Il semble pourtant qu'avec Achterberg l'articulation entre le vécu littéraire et le vécu biographique présente une intensité particulière. Ce rapport détermine le fonctionnement de la thématique : elle reçoit du poète sa structure mais la réciproque est également vrai. C'est peut-être là le point central de sa poétique. Il l'a développé comme thème sous la forme du couple permanent et pourtant jamais assuré, mais il s'y est également inscrit par sa pratique. Cette concentration absolue dont procède le suspens constaté dans des poèmes qui n'ont rien de flottant quant à la forme arrive à son point de rosée au moment où le poème "prend». Or ce moment ne s'intègre pas dans une réalité purement textuelle. Si elle a cette qualité d'événement, c'est que, pour le poète, il s'agit un accomplissement réel dont lui-même tire sa substance réelle. Tout se joue sur un outre passement des limites conventionnelles qui ordonnent les rapports entre le monde et l'individu et sur lesquelles la poésie moderne vient buter. Entendons donc R. Char lorsqu'il dit: « Les actions du poète ne sont que la conséquence des énigmes de la poésie. $»^{10}$

La levée du mystère entourant la vie d'Achterberg permet d'en avoir une vision d'ensemble. Sans prétendre, ce qui serait bien absurde, à quelque interprétation psychanalytique a posteriori et sans rabattre la biographie sur l'œuvre, il est possible de reconstituer quelques traits marquants des circonstances qui ont présidé à une très grande partie de l'œuvre. Dépasser l'horizon textuel du poème, c'est mettre en évidence les rapports structurés / structurants, selon les termes de J. Starobinski, dans lequel l'œuvre se déploie. Il est possible de dégager un ensemble de liens signifiants, qui, centrés sur l'œuvre, permettent de faire le départ entre le poète, construit par l'œuvre autant qu'il la construit, et la sphère privée qui est commune. L'identification ondoyante des images bibliques conduit à opérer la même séparation entre religion et foi. Celle-ci est adhésion personnelle, celle-là, basée sur le collectif, détourne du danger solipsiste. Elle sert le poème en l'ancrant dans un réel partagé, sinon partageable. La foi échappe à l'analyse dans la mesure où on ne peut la supposer différente du projet poétique.

L'année 1924 marque un tournant dans la vie de Gerrit Achterberg. Comme nous l'avons vu, il obtient un poste d'instituteur dans une école réformée, quitte son village natal avec sa communauté sociale et spirituelle soudée et abandonne également l'association Thimotheus dont il avait été un membre très actif. Cette même année il fait deux rencontres importantes: J.-A. Dekker qui va l'initier à la poésie et Cathrien van Baak avec laquelle il aura une relation mouvementée, intense et brève. Elle deviendra sa femme en 1946. En novembre de la même année, il publie, avec son ami, un recueil, De zangen van twee twintigers [Les chants de deux représentants de la génération de 1920], préfacé par le père de J. Dekker, qui est pasteur. Achterberg rejettera par la suite, sans la renier, cette œuvre de jeunesse qui manquait de maturité, mais il y était déjà question d'une horloge mesurant "la vie dans la mort». Tous les éléments qui façonneront le destin du poète sont déjà là, le reste n'est qu'affaire de logique.

Cette première période, qui se termine en 1937, est dominée par l'intensité avec laquelle il s'investit dans la poésie comme dans ses relations sentimentales. Le premier objet conduit à une désocialisation progressive. De perte d'emploi en perte d'emploi l'enseignement lui étant devenu insupportable - il devient fonctionnaire de $3^{\mathrm{e}}$ classe et risque de perdre tout statut ou ressource. Dès 1925, date à laquelle il commence son 
œuvre, il était évident pour lui que poésie et enseignement sont incompatibles : il faut choisir. Ses relations amoureuses, placées sous le signe de la violence, suivaient le même chemin. Sentant Cathrien, ou d'autres plus tard, lui échapper, il recourait aux menaces voire à la violence, obtenant évidemment l'effet inverse. Il acheta un pistolet, menaçait à l'occasion de se suicider. La police dut s'en mêler et il eut affaire aux milieux médicaux : en 1932 puis en 1933, il fut interné pour peu de temps. Peu avant, il avait publié un premier recueil Afvaart [Départ] (1931) d'une cinquantaine de poèmes qui pour une part avaient parus dans des revues à partir de 1926.

61 Cette trajectoire arrive en 1937 à une conclusion tragique. Installé dans un meublé, plus solitaire que jamais, Achterberg, dans un moment de panique dont les circonstances ne sont pas totalement éclaircies, tue sa logeuse d'un coup de revolver et blesse la fille de celle-ci, âgée de seize ans. Il se constituera rapidement prisonnier. Après six mois de détention préventive, la justice conclut à son irresponsabilité et prononce, pour protéger la société, une mesure de mise à disposition du gouvernement. En d'autres termes, Achterberg sera interné pour une durée indéterminée jusqu'à ce qu'une commission composée de psychiatres et de représentants du ministère décide de la guérison, ou du moins de l'innocuité du malade.

La mise à la disposition du gouvernement ne sera levée qu'en juin 1955, mais à partir de 1944 cette mesure fera l'objet d'assouplissements et le poète se trouvera placé dans une famille d'accueil. C'est dans ce contexte que se situe son mariage avec C. van Baak en 1946. L'année suivante, il recevra son premier prix littéraire: c'est le début d'une reconnaissance dépassant le petit groupe d'écrivains qui l'avait soutenu tout au long de ces années. Il obtiendra notamment, en 1950, le Prix national des lettres.

Le drame de 1937 constitue une traversée du miroir. La vie et l'œuvre se mêlent: prenant place dans la même continuité, l'une et l'autre se confirment et se renforcent. La critique y verra, un peu rapidement, l'origine et, du coup, l'explication de l'œuvre alors que le thème précède de plus de dix années les faits. Mais il s'agit de plus encore. Durant ces années d'internement et de surveillance étroite, Achterberg va produire la plus grande partie de son œuvre. De 1939 à 1949 il publiera $19^{11}$ recueils contre 6 de 1950 à sa mort en 1962. Le système dans lequel il est pris et dont personne par ailleurs ne conteste la nécessité même si ses amis tenteront d'améliorer sa situation, a donc eu un effet incontestable sur son travail poétique.

64 Le point sur lequel les commissions, qui ont eu à se prononcer sur la mise en liberté du poète, butaient sans cesse est l'absence de culpabilité, dont le poète ne faisait d'ailleurs pas mystère. Chacun a interprété ce fait en fonction de son point de vue philosophique ou religieux: l'homme est par essence coupable quoiqu'il fasse (calviniste), par son œuvre le poète tente de se racheter (catholique), le poète est libre et tout puissant dans son œuvre (laïc). La vérité semble plus simple : la mort qu'il a provoquée - pour laquelle la justice l'a déclaré irresponsable - est de la même nature que ses poèmes : absolue puisque structurant le réel et remédiable puisque l'effort peut et doit toujours être repris : tout dépend du poème à venir. Lorsqu'on lui demanda s'il ne ressentait pas de pitié envers la jeune fille qu'il a rendue orpheline, il répondit : « mais j'ai pourtant écrit cinq poèmes là-dessus $»^{12}$. Il faut donc revenir au poème, mais, pour ce faire, précisons d'abord les conditions ayant présidé à leur création.

65 Le dispositif dans lequel sera pris Achterberg durant ces années si productives se caractérise par trois aspects fondamentaux : la soumission à la commission qui a tous les pouvoirs sur le poète ; l'enfermement; l'absence de public, c'est-à-dire de 
reconnaissance officielle. Ces trois contraintes se renforcent mutuellement et expliquent l'importance, a priori inattendue, que prend le fait de ne pas recevoir un prix qui aurait été un premier pas vers un accueil plus large :

En het is voor mij, alsof het al of niet verwerven van de Van der Hoogtprijs m'n verzen staan of vallen. Dan is er voor de dood in het leven geen plaats en moet ik, logisch gesproken, verdwijnen. ${ }^{13}$

[Et c'est pour moi comme si par le fait d'obtenir ou non le prix Van der Hoogt mes poèmes existent ou pas; Alors il n'y a pas de place pour la mort dans la vie et je devrai, logiquement, disparaître.] rencontre à partir d'une nécessité intérieure. C'est alors que le thème central va se cristalliser dans toute sa complexité. Après 1937, ce rapport au public, instance de reconnaissance légitimant la réalité de sa poésie, va se poser en termes de survie. L'autorité tentaculaire de la commission formule clairement une attente d'intégration sociale s'appliquant également à la poésie: faire acte de contrition, accepter les conventions. La force créatrice qui anime le poète s'y refuse absolument, en est incapable. Achterberg est donc pris dans une alternative sans solution : être anéanti ou s'anéantir soi-même ; d'où la persistance des tendances suicidaires. Cette situation sans issue s'exprime notamment dans une stratégie paradoxale de dédicaces. Le poète dédie, avec l'espoir avoué d'améliorer "son cas", recueils et poèmes aux détenteurs du pouvoir, psychiatres, directeurs ou fonctionnaires ministériels. Ces œuvres trouvent effectivement leur place dans son dossier, mais généralement comme pièces à charge. 
70 Cette alternative impossible, qui menace l'existence même du poète, trouve dans la poésie une solution. Celle-ci passe par la constitution d'une instance intérieure qui prend en charge l'œuvre et permet sa concrétisation par un accueil sans réserve ${ }^{14}$. Cette instance présente deux caractéristiques fondamentales qui se trouvent clairement inscrites dans l'œuvre.

71 La première est le caractère extrêmement variable que peut prendre cette figure intérieure. Sa fonction est précisément d'accepter toutes les formes de pulsions, toutes les projections. C'est cette malléabilité qui donne son unité à la constitution de l'œuvre. Celle-ci est la partie visible d'un objet dont le centre de gravité est peut-être ailleurs. Le passage sans solution de continuité du Christ à la femme aimée, puis au poème, suppose moins un commun dénominateur thématique qu'elle n'implique la permanence d'une structure de base. D'où également la facilité avec laquelle le Je adopte les deux sexes «Ik ben een man geworden met twee lijven, / ni. dat van mij en van mijn vrouw» [Je suis devenu un homme avec deux corps, / c.-à-d. le mien et celui de ma femme] («Sexoïde ») -, en change ou encore adopte des formes diverses comme dans le célèbre poème «De dichter is een koe» [Le poète est une vache]. Il est cependant évident que la forme principale, ce Vous dans laquelle on a vu une nouvelle Eurydice, prévaut pour des raisons biographiques. Les poèmes des premières années, notamment les poèmes érotiques dont le caractère, pourtant affirmé, échappait au jeune poète qui s'étonnait des réactions suscitées, s'appuyaient sur des expériences réelles. Mais aussi bien la réalité résiste à l'assimilation poétique et il est naturel que tout en conservant un substrat historique, cette figure soit absorbée par l'instance intérieure. La conquête du Vous sur la mort, dont nous avons vu le caractère de présence immanente et insaisissable, pourrait être une transposition de ce processus où la femme réelle, objet d'amour et de violences, doit mourir au réel pour être capté par le poème. D'où le caractère positif de cette présence dans le poème se constituant et non dans l'œuvre faite sinon à l'état de traces : il s'agit d'un processus, d'une action se déployant dans le monde.

Ce caractère processuel implique également que le poème en tant que tel, même si, dans sa perfection il est la condition sine qua non pour mener à terme le mouvement, n'est pas l'unité de base. Achterberg est habité par la poésie: «j'ai neuf nouveaux poèmes (pas sur le papier, mais dans la tête). Ils pourraient être neuf mille. (Ce qui ne veut rien dire non plus d'ailleurs.) » ${ }^{15}$. L'élaboration psychique et la création poétique vont de pair. Pas plus que dans cette élaboration le temps n'a de réalité, le poète n'accordait d'importance à la chronologie dans la publication de ses poèmes; à tel point que la date de première parution est souvent la seule indication, et elle n'est guère significative, pour suivre la progression de l'œuvre.

73 La seconde caractéristique concerne les bases sur lesquels la création poétique fait fond. Le caractère prégnant de l'effet de réel que dégage la poésie d'Achterberg pourrait s'expliquer par la nature des forces engagées. Celles-ci, par les identifications primitives qu'elles supposent, plongent au plus profond de l'inconscient. La constitution de l'œuvre met ainsi directement en œuvre la libido sublimée, tirant son énergie $\mathrm{du}$ ça et exerçant sa tendance à organiser l'informe, à produire de la structure $^{16}$. Cet engagement de l'être psychique tout entier est rendu possible par un retrait du moi, instance de régularisation interne constituant la relation avec le réel, renforcé par l'état d'apesanteur psychique dans lequel se trouve le poète pendant son 
internement. C'est là, sans doute, que joue le plus nettement le phénomène de résonance produit par l'homologie entre intérieur et extérieur.

Ainsi le poète devient réellement cette pure attente du poème à venir. Il sent les forces agir sans que le Je y ait part. Dans «Zondag» - un des poèmes consacrés à l'internement, rassemblés par l'auteur, et publiés après sa mort en 1969 dans le recueil Blauwzuur - il pose le problème du rapport au réel : «Waarom leg ik dus mijn pen niet weg / en sterf liever, dan in te gaan / op de verlokking van droom en waan?» [Pourquoi est-ce que je ne pose donc pas mon stylo / et préfère mourir, plutôt que de répondre / aux avances du rêve et de l'illusion ?] La réponse est donnée dans les deux vers de la strophe finale : «Een bladstil overgebogen vlam / beheerst het plan buiten mij om.» [Un flamme immobile, recourbée / domine le projet en dehors de moi.] On trouve dans ces vers à la fois le caractère compulsif de l'écriture poétique et la maîtrise de l'homme de la pratique qu'est tout créateur.

Il existe un déséquilibre profond entre l'investissement exclusif dans le processus poétique et le désinvestissement dont la réalité est frappée. Les pulsions, qui auparavant s'exprimaient de façon parfois violente, se trouvent à présent mobilisés par le projet poétique. Celui-ci remplace en partie la mise en adéquation avec le monde sans qu'il soit question d'une véritable déréalisation, même si le risque existe. La concrétisation de l'œuvre, ses contraintes formelles, la résistance du matériau linguistique, la nécessité de publier enfin, établissent un point de repère extérieur. On comprend alors l'ambivalence avec laquelle il aborde la publication. La face sociale du poème du poème lui est étrangère : "C'est une affaire entre moi et le poème. C'est tout. $\aleph^{17}$ II aura toujours un ami, conseillé et secrétaire à la fois, à qui il déléguera ce travail, y compris souvent la composition des recueils ou la recherche de titres manquants. Mais le poème a également besoin d'une confirmation extérieure. N'ayant pas de position littéraire à défendre, et moins encore à imposer, Achterberg doit trouver des relais assurant une réception minimale garantissant la validité du poème, son insertion dans un milieu, même restreint. Cette dépendance vis-à-vis du lectorat répond à la nature du processus poétique intérieure et, d'un certain point de vue, en fait partie. L'accession au monde extérieure prouve la réalité de l'ensemble. La phrase qui accompagne la plupart de ses envois, "Ce qui n'est pas bon, n'est pas écrit », doit être prise littéralement.

L'évolution de l'œuvre montre l'importance de la forme. Le changement principal que l'on peut repérer, et qui est surtout marqué dans les derniers recueils, est l'importance de plus en plus grande accordée aux techniques de la poésie et à l'élaboration complexe d'enchaînements symboliques. Il compose plusieurs cycles fermement structurés ${ }^{18}$ et le sonnet devient sa forme de prédilection. De plus, à la faveur des rééditions et de la publication de la première version de ses œuvres complètes (Cryptogamen I à IV, 1960-61) il reprendra certains vers pour en améliorer le rythme.

Mais pour arriver à ce poème-objet réel, le moi passe par une phase d'effacement face au destinataire intérieur, au Vous si puissant, à la poésie. Durant cette phase le sens de l'individualité tend à se dissoudre, la frontière avec le réel s'estompe :

Laat mij...

Laat mij $\mathrm{u}$ tot een lied herleiden,

dat er in zal geborgen zijn,

uw nameloos verblijden,

opdat het zal voorhanden zijn 
als ik niet meer kan onderscheiden

wie ik tussen de mensen ben.

Laissez-moi...

Laissez-moi vous réduire à un chant, qui sera dedans en sûreté, votre jubilation sans nom, afin qu'elle soit disponible lorsque je ne saurai plus distinguer qui je suis parmi les gens. exceptionnel de clairvoyance $»^{19}$. Chez Achterberg cela prend la forme d'une rare capacité à lier par association les éléments les plus divers qui, pris dans les rets de son inspiration, acquièrent un caractère d'évidence. L'espace référentiel se courbe sous l'attraction exercée par une nécessité qui ne reconnaît pas le réel en tant que tel. Les linéaments parallèles du sens s'incurvent et convergent vers un même point. Dans le mouvement de dissolution - recomposition la tension qui fait le poème prime le matériau tout comme le poème à venir annule tout ce qui précède. Là se trouve sans doute un élément d'explication de la fascination que produit cette poésie. Chaque image, chaque phrase portent la marque de l'évidence, mais c'est toujours autre chose qui est à l'œuvre.

Les éléments religieux ne sont donc qu'une partie d'un vaste ensemble d'images, de symboles et de significations. Moïse ou le Christ ne sont que le support d'un même effort de structuration, tout comme, par exemple, l'osmose : «L'osmose tend à rendre égales les concentrations moléculaires des deux solutions (-Isotonie), l'eau passant de la solution faible à la solution plus forte. " (Le Robert). L'importance d'un symbole ne tient donc pas à quelque vertu intrinsèque mais à sa conductibilité qui, créant des liens, organise l'informe. Pour cette raison le destinataire est réduit à une pure forme pronominale, véritable point aveugle linguistique.

81 L'importance de la thématique religieuse n'a d'autres limites que la perspicacité du lecteur. De l'emprunt aux Ecritures à la rémanence de rythmes caractéristiques des Psaumes versifiés, voire de cadence de chants d'église, nombreux sont les passages relevés par une critique essentiellement calviniste. Il y a là évidemment une culture commune. Mais ce regard particulier porte en lui-même sa propre limite puisqu'il implique l'idée de définition - est-il calviniste, est-il chrétien? Le même phénomène joue sur un plan purement littéraire et la question de savoir si l'auteur appartient au romantisme, au symbolisme ou à la modernité reste sans réponse.

82 Le processus de création utilise les traces du passé de l'auteur pour constituer la figure nouvelle. L'importance de la religion est donc à la mesure de la place qu'elle occupa dans la vie de l'auteur jusqu'en 1924. Mais il y a plus important. Le calvinisme met très fortement l'accent sur le Verbe et cette insistance est reprise et renforcée encore par la 
religion réformée hollandaise qu'Achterberg a connue sous une forme rigoureuse et dont il est profondément imprégné. Bien plus que l'image, sa "primitive passion » est le mot. Du logos redéfini au fil des siècles procède une conception de l'homme et de son rapport au monde. Achterberg puise à cette source par l'intermédiaire de l'interprétation chrétienne de la parole, essentiellement celle de Jean, le plus philosophe des évangélistes, et qu'il s'approprie en tant que poète. Parole et poème transforment l'abîme en un monde accessible à l'homme, qui exerce ainsi sa capacité créatrice. L'élément religieux dans la poésie d'Achterberg conduit à placer la parole au centre de l'humain. Au plus profond de sa douloureuse expérience, dont il n'a pas été la seule victime, il fait l'expérience de la dimension fondatrice de la parole. Le processus psychique ouvre sur l'ontologie si, répondant au poème, le texte se déclôt. Cette expérience est toujours particulière et n'appartient qu'au langage en acte. C'est la raison pour laquelle Achterberg, homme de la pratique, parle toujours du poème, rarement de poésie. La rencontre entre être et existence est toujours singulière.

En opposant, dans la deuxième Epître aux Corinthiens, la lettre et l'esprit, Paul ne dit pas autre chose. Le poète s'approprie l'image en préservant le schème fondamental commun à la religion et à la poésie. Le poème a la capacité de préserver la vie contre la chaos et d'accomplir l'incarnation du sens. Achterberg est parfaitement conscient de ce rapport détourné au Livre :

Men zegt, dat ik christelijke Symbolen en figuren voor particulier gebruik beschouw. Natuurlijk! Anders betekent het toch niets! ${ }^{20}$

[On dit que je prends des symboles et des personnages chrétiens pour un usage personnel. Mais bien sûr ! Sinon cela ne veut rien dire !]

La Bible n'est qu'une des voies pour créer le poème et en retrouver le principe. De thème en image, elle a accompagné le mouvement d'un individu vers l'assomption de son essence poétique. L'œuvre est la réalisation de cette intuition fondamentale: l'homme et le monde sont coextensifs et le poème a accès au réel.

\section{NOTES}

1. - L'expression est de J. Bogerman (1576-1637) qui a joué un rôle éminent dans la réalisation de la nouvelle traduction.

2. - Les éléments biographiques sont tirés de l'ouvrage de référence : W. Hazeu, Gerrit Achterberg, een biographie, $3^{\mathrm{e}}$ édition augmentée. De Arbeiderspers, 1989.

3. - Les citations françaises de la Bible sont faites selon l'édition d'Edouard Dhorme, Gallimard, coll. de la Pléiade, 3 vol., Paris, 1956.

4. - Tous les poèmes sont tirés des œuvres complètes : G. Achterberg, Verzamelde gedichten, Em. Querido, Amsterdam, 10éd., 1988, 1061 pages.

5. - Les traductions ne sont que des transcriptions vers à vers afin d'aider le lecteur à suivre l'original.

6. - R.L.K. Fokkema, Varianten bij Achterberg, 2 vol., Em. Querido, Amsterdam, 1980.

7. - Y. Bonnefoy, Entretiens sur la poésie, A la Baconnière, Neuchatel, 1981, p. 158. 
8. - E. Benveniste, «De la subjectivité dans la langue », Problèmes de linguistique générale, 2 vol., Gallimard, coll. Tel, Paris, 1976. vol. 1, p. 259.

9. - G. Achterberg, Briefwisseling met zijn uitgevers [Correspondance avec ses éditeurs], 2 vol., Em. Querido, Amsterdam, 1989, vol. 1, p. 257.

10. - R. Char, Recherche de la base et du sommet, "A une sérénité crispée », in : Euvres complètes, Gallimard, Paris, 1983, p. 753.

11. - Avec En Jezus schreef in het zand (1947) qui reprend certains poèmes déjà parus en recueil et Blauwzuur (Acide prussique) composé de poèmes ayant pour thème l'asile et rassemblés par l'auteur, mais publié après sa mort en 1969. Cela représente près de 700 poèmes repris dans les Euvres complètes, mais sa production fut sans doute bien plus importante.

12. - Cité par W. Hazeu, Op. cit., p. 215.

13. - Lettre au poète Ed. Hoornik, 5 mai 1940, in: G. Achterberg, Briefwisseling met Ed. Hoornik, Em. Querido, Amsterdam, 1990, p. 38.

14. - Cf. M. de M’Uzan, "Aperçus sur le processus de la création littéraire ", De l'art à la mort, Gallimard, Paris, 1977.

15. - Lettre au poète Ed. Hoornik, 22 mars 1941, in: Op. cit., p. 53. «ik heb negen nieuwe verzen (niet op papier, maar in mijn hoofd). Het kunnen er negenduizend worden (Dat zegt trouwens ook niets).»

16. - Cf. S. Freud, « Le moi et le ça », trad. J. Laplanche, in Essais de psychanalyse, Payot, Paris, 1981. 17. - Lettre à Ed. Hoornik, 30 novembre 1940. Op. cit., p. 43. «Het is een kwestie tusschen mij en het vers. Dat is alles.»

18. - Notamment : Ballade van de gasfitter [La ballade de l'ouvrier du gaz], 1953 ; Spei van de wilde jacht [Le jeu/représentation de la chasse sauvage], 1957.

19. - M. de M’Uzan, Op. cit., p. 26.

20. - Cité par S. Dresden, «Horizontale poëzie» in: B. Bakker et A. Middeldorp réd., Nieuw commentaar op Achterberg, Bert Bakker, Daamen n.v., La Haye, 1966. p. 14.

\section{RÉSUMÉS}

Aborder une œuvre sous l'angle de la religion et de la foi conduit à dépasser le problème des thèmes pour saisir l'œuvre dans un ensemble signifiant plus large. Le poème ne se réduit pas au texte. Fortement marqué durant sa jeunesse par le calvinisme, le poète Gerrit Achterberg (1905-1962) intègre la Parole dans son œuvre où citations, thèmes, figures et lieux bibliques abondent. Bien loin de se révolter contre cette emprise, il constituera même un recueil destiné au public protestant.

Pourtant, même dans les poèmes les plus orthodoxes, se fait jour un glissement par lequel Dieu, le Christ et le poème finissent par se confondre avec la femme aimée, destinataire emblématique de l'œuvre. Le caractère hétérogène de la thématique souligne, plus qu'elle ne cache, une profonde unité au sein de laquelle l'élément religieux joue un rôle particulier.

Pris dans un rapport structurant réciproque, poème et poète constituent un système dans lequel les éléments épars prennent un caractère de nécessité et où la poésie occupe tout l'espace. Du fait religieux ne reste qu'une structure fondamentale. Si, du point de vue thématique, la Bible n'est qu'une source parmi d'autres (encyclopédie, manuels divers etc.), la conception chrétienne de la Parole, surtout celle de Jean, ouvre la voie vers une définition de l'homme et du monde qui fonde 
la capacité créatrice de la poésie. La place centrale de la parole rétablit le lien entre l'élaboration psychique et la question de l'être. Détachée de la religion, l'œuvre réalise cette intuition fondamentale : l'homme et le monde sont coextensifs et le poème a accès au réel.

Een literair werk met als invalshoek godsdienst en geloof bestuderen brengt met zieh mee dat de thematiek binnen een ruimer verband moet worden geplaatst. Een gedieht is niet louter tekst. Het calvinisme heeft een diepe invloed gehad op de jonge Gerrit Achterberg (1905-1962). Het Woord is in zijn werk aanwezig en bijbelse thema's, figuren en citaten komen er veelvuldig in voor. Niet alleen heeft hij er zieh niet tegen verzet maar hij heeft zelfs een bundel samengesteld voor de protestante lezers.

Ook in zijn meest orthodoxe gedichten blijkt er een verschuiving plaats te vinden waardoor God, Christus et het gedieht samen vallen met de geliefde, het bekende centrale thema. De zeer uiteenlopende thematiek maakt de fundamentele eenheid van het werk juist duidelijk. Het godsdienstig element speelt hierbij én aparte rol.

Door hun structurerende kracht vormen de dichter en de gedichten samen een systeem waarin willekeur en verdeeldheid door noodzakelijkheid worden verbonden en de poëzie overal aanwezig is. Van het godsdienstige blijft slechts én fundamenteel schéma over. De Bijbel is, op thematisch vlak, een van de vele bronnen naast de encyclopédie e.d., maar de christelijke opvatting van het Woord, vooral bij Johannes, maakt het mogelijk om tot een definitie van mens en wereld te komen die de scheppende kracht van de poëzie kan grondvesten. Doordat het woord een centrale plaats krijgt kan men tot de eenheid komen van het psychisch proces en het zijn. Los van godsdienst verwezenlijkt het werk deze fundamentele intuïtie: mens en wereld bezitten een gelijksoortige strekking en het gedieht heeft toegang tot de werkelijkheid.

\section{AUTEUR}

\section{SPIROS MACRIS}

Université Charles-de-Gaulle Lille III 